\title{
Qualidade física e frações da matéria orgânica de um Planossolo sob sistema plantio direto
}

\author{
Diony Alves Reis ${ }^{(1)}$, Claúdia Liane Rodrigues de Lima ${ }^{(2)}$ e Adilson Luís Bamberg ${ }^{(3)}$
}

\begin{abstract}
(1)Instituto Federal de Educação, Ciência e Tecnologia do Rio Grande do Sul, Avenida Osvaldo Aranha, no 540, Juventude da Enologia, CEP 95700-000 Bento Gonçalves, RS, Brasil. E-mail: dionyodin@gmail.com (2)Universidade Federal de Pelotas, Faculdade de Agronomia Eliseu Maciel, Campus Universitário, s/nº, Caixa postal 354, CEP 96010-900 Pelotas, RS, Brasil. E-mail: clrlima@yahoo.com.br (3)Embrapa Clima Temperado, Rodovia BR 392, Km 78, Caixa Postal 403, CEP 96010-971 Pelotas, RS, Brasil. E-mail: adilson.bamberg@embrapa.br
\end{abstract}

Resumo - O objetivo deste trabalho foi avaliar a qualidade física e as frações da matéria orgânica de um Planossolo sob sistema plantio direto (SPD), com diferentes tempos de implantação, no Sul do Brasil. Amostras de solo foram coletadas nas camadas de $0,00-0,03,0,03-0,06,0,06-0,10$ e $0,10-0,20 \mathrm{~m}$, tendo-se determinado: a porosidade total $(\mathrm{Pt})$; a macroporosidade $(\mathrm{Ma})$; a microporosidade $(\mathrm{Mi})$; a resistência do solo à penetração $(\mathrm{Rp})$; a densidade (Ds); os macro e microagregados; o diâmetro médio ponderado dos agregados (DMP); o carbono orgânico total (COT); o carbono das frações leve livre (FLL), leve oclusa (FLO) e pesada (FP); os estoques de carbono (EstC), os índices de labilidade (ILC), de estoque (IEC) e de manejo do carbono (IMC). O tempo de implantação SPD aumentou a Pt, a Ma, os EstC, e diminuiu a Mi, a Rp e a Ds. O SPD promove os atributos DMP, ILC, IEC e IMC a partir do quinto ano de implantação, e, portanto, melhora a qualidade física do solo.

Termos para indexação: dinâmica da matéria orgânica, estruturação do solo, fracionamento da matéria orgânica, índices de labilidade, solo hidromórfico, solos de várzea.

\section{Physical quality and organic matter fractions of an Alfisol under no-tillage}

\begin{abstract}
The objective of this work was to evaluate the physical quality and the organic matter fractions of an Alfisol soil under no-tillage (NT) with different deployment times in Southern Brazil. Soil samples were taken at the $0.00-0.03,0.03-0.06,0.06-0.10$, and $0.10-0.20-\mathrm{m}$ depths, for determining: total porosity (Pt); macroporosity (Ma); microporosity (Mi); soil penetration resistance (Rp); bulk density (Ds); macro- and microaggregates; mean weight diameter (MWD) of soil aggregates; total organic carbon content (TOC); the free light (FLL), light occluded (FLO), and heavy (FP) carbon fractions; carbon stocks (EstC); and the lability (ILC), stock (IEC), and carbon management (IMC) indexes. SPD deployment time increased Pt, Ma, and EstC, and decreased Mi, RP, Ds. NT promotes MWD, ILC, IEC, and IMC after five years of deployment, and, therefore, it improves soil physical quality.
\end{abstract}

Index terms: organic matter dynamics, soil structuration, organic matter fractioning, lability indexes, hydromorphic soil, lowland soils.

\section{Introdução}

Os solos de várzea no Rio Grande do Sul compreendem 5,4 milhões de hectares ou $20 \%$ da área do estado (Gomes et al., 2006), que têm sido cultivados com arroz irrigado sob diferentes sistemas de manejo. Dessa área, um milhão de hectares têm sido ocupados anualmente com a cultura, enquanto o restante tem sido utilizado com pecuária de corte extensiva ou mantido sob pousio. Apesar da evolução da área plantada com culturas de sequeiro sob sistema plantio direto (SPD) na região, o emprego do preparo convencional (PC) (Irga, 2014) ainda tem limitado a sustentabilidade da produção agrícola e resultado na degradação do solo, além dos elevados custos de preparo (Vernetti Júnior et al., 2009).

Assim como nas áreas de terras altas, os solos de várzea podem apresentar redução nos teores de matéria orgânica, erosão superficial e compactação devida ao manejo (Lisboa et al., 2012). Ademais, a degradação do solo tem sido relatada como consequência da ação antrópica, sobretudo das práticas agrícolas, o que evidencia a necessidade de utilização de alternativas mitigadoras da compactação e da desestruturação desses solos; entretanto, poucos trabalhos têm objetivado avaliar a qualidade física e as frações da matéria orgânica de solos de várzea. 
A separação das frações físicas da matéria orgânica do solo (MOS), com base na diferença de densidade entre a fração leve livre (FLL) e a fração pesada (FP) (Conceição et al., 2008), tem possibilitado a criação de índices para avaliação da qualidade física do solo. Nesse sentido, Blair et al. (1995) sugeriram o índice de manejo de carbono (IMC) como adequado para a síntese de aspectos qualitativos (labilidade ou suscetibilidade à alteração pelo sistema de manejo e microrganismos) e quantitativos (estoque de carbono) da MOS, comparados a uma condição de referência (IMC $=100 \%$ ), geralmente uma área de vegetação nativa.

O objetivo deste trabalho foi avaliar a qualidade física e da matéria orgânica de um Planossolo, com diferentes tempos de implantação do sistema de plantio direto.

\section{Material e métodos}

O estudo foi realizado na Estação Experimental de Terras Baixas, pertencente à Embrapa Clima Temperado, no Município de Capão do Leão, Rio Grande do Sul. As coordenadas da estação são 3149'04,13"S e 52²7'53,77"O. Conforme a classificação de Köppen, o clima da região é do tipo Cfa, mesotérmico quente, com média do mês mais frio entre 3 e $18^{\circ} \mathrm{C}$, precipitação pluvial média mensal não inferior a $60 \mathrm{~mm}$, sempre úmido, e a temperatura do mês mais quente é superior a $22^{\circ} \mathrm{C}$. $\mathrm{O}$ solo foi classificado como Planossolo Háplico eutrófico típico, de textura franca ( $460 \mathrm{~g} \mathrm{~kg}^{-1}$ de areia, $370 \mathrm{~g} \mathrm{~kg}^{-1}$ de silte e $170 \mathrm{~g} \mathrm{~kg}^{-1}$ de argila) até a profundidade de $0,20 \mathrm{~m}$ (Cunha \& Costa, 2013).

Historicamente, as áreas avaliadas eram manejadas sob preparo convencional (PC), e foram homogeneizadas antes da implantação do SPD por meio de subsolagem e correção da acidez (Manual..., 2004), mediante incorporação superficial de calcário dolomítico com grade de discos e pelo estabelecimento de diferentes plantas de cobertura (Tabela 1), que receberam $300 \mathrm{~kg} \mathrm{ha}^{-1}$ de fertilizante mineral da fórmula $\mathrm{N}-\mathrm{P}_{2} \mathrm{O}_{5}-\mathrm{K}_{2} \mathrm{O}$ 2-20-20, para leguminosas de verão, e $300 \mathrm{~kg} \mathrm{ha}^{-1}$ de 5-20-20 na base $+100 \mathrm{~kg} \mathrm{ha}^{-1} \mathrm{de}$ $\mathrm{N}$ em cobertura, para gramíneas de verão e de inverno. Plantas de cobertura de inverno (azevém) receberam $100 \mathrm{~kg} \mathrm{ha}^{-1}$ de $\mathrm{N}$ em cobertura, enquanto as plantas espontâneas não receberam adubação.

O estudo compreendeu a comparação de áreas com 1 (SPD1), 3 (SPD3), 5 (SPD5) e 7 anos de implantação do sistema plantio direto (SPD7), e teve como referência uma área de campo não cultivado $(\mathrm{CN})$ por mais de 30 anos, ocupado com espécies espontâneas, não pastejado e adjacente às demais áreas.

A amostragem do solo foi realizada entre os meses de agosto e outubro de 2013 nas camadas de 0,00 a $0,03 \mathrm{~m}$; de 0,03 a $0,06 \mathrm{~m}$; de 0,06 a $0,10 \mathrm{~m}$ e de 0,10 a $0,20 \mathrm{~m}$. A escolha dessas camadas foi determinada pela suscetibilidade às alterações físico-hídricas decorrentes do sistema de manejo, dos sistemas radiculares das culturas adotadas e da proximidade com o horizonte B plânico do Planossolo $(\sim 30 \mathrm{~cm})$.

Amostras de solo com estrutura preservada foram coletadas em anéis volumétricos de $4,8 \mathrm{~cm}$ de diâmetro por 3,0 cm de altura, no total de 240 amostras (3 anéis por camada x 4 camadas de solo x 4 repetições x 5 tratamentos). Além disso, 80 amostras com estrutura não preservada foram coletadas ( 1 amostra 4 camadas de solo $\times 4$ repetições $\times 5$ tratamentos), as quais foram destorroadas manualmente em seus pontos de fraqueza e secas ao ar, em laboratório.

Amostras com estrutura preservada foram saturadas por capilaridade durante 48 horas para a determinação da porosidade total $(\mathrm{Pt})$, macroporosidade $(\mathrm{Ma})$, microporosidade (Mi) e relação $\mathrm{Mi}: \mathrm{Ma}$, tendo-se utilizado uma sucção de coluna de água de $0,60 \mathrm{~m}$

Tabela 1. Esquema de sucessão de culturas utilizado no Planossolo em estudo, sob diferentes tempos de implantação do sistema plantio direto.

\begin{tabular}{lcccccccc}
\hline \multirow{2}{*}{ Tratamento } & \multicolumn{7}{c}{ Ano agrícola } \\
\cline { 2 - 8 } & $05 / 06$ & $06 / 07$ & $07 / 08$ & $08 / 09$ & $09 / 10$ & $10 / 11$ & $11 / 12$ \\
\hline SPD1 & $\ldots$ & $\ldots$ & $\ldots$ & $\ldots$ & $\ldots$ & $\ldots$ & $\mathrm{Mi} / 13$ \\
SPD3 & $\ldots$ & $\ldots$ & $\ldots$ & $\ldots$ & $\mathrm{Mi}$ & $\mathrm{Az}+\mathrm{Es}+\mathrm{Sj}$ & $\mathrm{Tr}+\mathrm{Sj}$ & $\mathrm{Tr}+\mathrm{Sj}$ \\
$\mathrm{SPD} 5$ & $\ldots$ & $\ldots$ & $\mathrm{Mi}$ & $\mathrm{Az}+\mathrm{Es}+\mathrm{Sj}$ & $\mathrm{Tr}+\mathrm{Sg}$ & $\mathrm{Az}+\mathrm{Es}+\mathrm{Sj}$ & $\mathrm{Tr}+\mathrm{Sj}$ & $\mathrm{Az}+\mathrm{Es}+\mathrm{Sj}$ \\
SPD7 & $\mathrm{Mi}+\mathrm{Az}+\mathrm{Tb}$ & $\mathrm{Sj}+\mathrm{Tr}$ & $\mathrm{Mi}$ & $\mathrm{Tr}+\mathrm{Gs}$ & $\mathrm{Tr}+\mathrm{Mi}$ & $\mathrm{Az}+\mathrm{Es}+\mathrm{Sg}$ & $\mathrm{Az}+\mathrm{Es}+\mathrm{Sj}$ & $\mathrm{Az}+\mathrm{Es}+\mathrm{Sj}$ \\
\hline
\end{tabular}

SPD1, SPD3, SPD5 e SPD7 representam áreas com 1, 3, 5 e 7 anos de implantação do sistema plantio direto, respectivamente. Mi, milho; Tr, trigo; Az, azevém; Es, espontâneas; $\mathrm{Sj}$, soja; $\mathrm{Sg}$, sorgo; Tb, trevo branco; e Gs, girassol. 
(Claessen, 1997). Após a saturação e equilíbrio a $10 \mathrm{kPa}$ em câmara de pressão de Richards (Klute, 1986), foi determinada a resistência do solo à penetração $(\mathrm{Rp})$ com uso de um penetrômetro eletrônico de bancada (MA 933), dotado de variador eletrônico de velocidade e sistema de registro de dados (Tormena et al., 2007). As amostras foram secas em estufa a $105^{\circ} \mathrm{C}$, até estabelecimento de massa constante, para obtenção da densidade do solo (Ds) (Claessen, 1997).

As amostras com estrutura não preservada foram utilizadas para determinação dos macroagregados, microagregados e diâmetro médio ponderado dos agregados estáveis em água (DMP), de acordo com o procedimento de Kemper \& Rosenau (1986), modificado por Palmeira et al. (1999), com uso de equipamento de oscilação vertical (Yoder, 1936) e diâmetro de agregados de $0,25 \mathrm{~mm}$ como limite entre macro e microagregados (Tisdall \& Oades, 1982).

Agregados passados em malha de $9,51 \mathrm{~mm}$ foram utilizados no fracionamento densimétrico da MOS, tendo-se utilizado $10 \mathrm{~g}$ de solo em $80 \mathrm{~mL}$ de solução de politungstato de sódio (PTS), com densidade de $2,00 \mathrm{~g} \mathrm{~cm}^{-3}$. Na centrífuga, foram obtidas as frações leve livre, leve oclusa e pesada (FLL, FLO, FP, respectivamente) da MOS (Conceição et al., 2008). O conteúdo de COT e das frações foi determinado por combustão seca, via analisador elementar PerkinElmer.

Os estoques de COT foram calculados pela massa equivalente (Sisti et al., 2004), tendo-se utilizado a densidade média em cada camada de solo e o CN como referência, conforme a equação:

$$
\mathrm{Cs}=\sum_{\mathrm{i}=1}^{\mathrm{n}-1} \mathrm{C}_{\mathrm{ti}}+\left[\mathrm{M}_{\mathrm{tn}}-\left(\sum_{\mathrm{i}=1}^{\mathrm{n}} \mathrm{M}_{\mathrm{Ti}}-\sum_{\mathrm{i}=1}^{\mathrm{n}} \mathrm{MS}_{\mathrm{i}}\right)\right] \mathrm{C}_{\mathrm{tn}}
$$

em que Cs corresponde ao estoque de $\mathrm{C}$ corrigido em função da massa de solo da área de referência $\left(\mathrm{Mg} \mathrm{ha}{ }^{-1}\right) ; \sum_{\mathrm{i}=1}^{\mathrm{n}-1} \mathrm{C}_{\mathrm{Ti}}$ é a soma do COT $\left(\mathrm{Mg} \mathrm{ha}^{-1}\right)$ da camada superficial à penúltima camada (n-1) do tratamento avaliado; $\mathrm{M}_{\mathrm{tn}}$, a massa do solo da última camada amostrada no tratamento $\left(\mathrm{Mg} \mathrm{ha}^{-1}\right) ; \sum_{\mathrm{i}=1}^{\mathrm{n}} \mathrm{M}_{\mathrm{ti}}$, a soma da massa de solo $\left(\mathrm{Mg} \mathrm{ha}^{-1}\right)$ na camada superficial (1) até a camada mais profunda (n) no tratamento avaliado; $\sum_{\mathrm{i}=1}^{\mathrm{n}} \mathrm{MS}_{\mathrm{i}}$, o somatório da massa total do solo amostrado na área de referência $\left(\mathrm{Mg} \mathrm{ha}^{-1}\right)$, e $\mathrm{C}_{\mathrm{tn}}$, o teor de $\mathrm{C}$ do solo na última camada amostrada $\left(\mathrm{Mg} \mathrm{Mg}^{-1}\right.$ de solo).
Antes da correção pela massa de solo, os estoques de $\mathrm{C}$ de cada camada, em todas as áreas, foram calculados pela equação de Veldkamp (1994): EstC $=($ COT $\times$ Ds $\times$ e $) / 10$; em que EstC é o estoque de COT total em determinada profundidade $\left(\mathrm{Mg} \mathrm{ha}^{-1}\right)$; COT, o teor de COT na profundidade amostrada $\left(\mathrm{g} \mathrm{kg}^{-1}\right)$; Ds, a densidade do solo da profundidade avaliada $(\mathrm{Mg}$ $\mathrm{m}^{-3}$ ) e e, a espessura da camada considerada (m). A labilidade do carbono (LC) foi calculada pela razão entre a fração de carbono lábil e a fração de carbono não lábil, de acordo com: $\mathrm{LC}=\mathrm{C}$ lábil / $\mathrm{C}$ não lábil; em que $\mathrm{C}$ lábil é o estoque de carbono da fração leve (FLL + FLO) e C não lábil é o estoque de carbono da FP. O IMC, o IEC e o ILC foram calculados para a camada de 0,00 a $0,20 \mathrm{~m}$, tendo-se considerado como referência o $\mathrm{CN}(\mathrm{IMC}=100 \%)$, conforme Blair et al. (1995): IMC $=$ IEC $\times$ ILC $\times 100$, em que IEC é o estoque de $\mathrm{C}$ do tratamento dividido pelo estoque de $\mathrm{C}$ da referência, e o ILC é a labilidade do carbono no tratamento considerado, dividido pela labilidade do carbono da referência (Blair et al., 1995).

A normalidade dos dados foi verificada pelo teste de Shapiro-Wilk (W) $(\mathrm{n} \leq 200)$. Valores discrepantes foram identificados e excluídos por meio de medidas do limite inferior (LI) e do limite superior (LS), tendo-se considerado o primeiro quartil (Q1), o terceiro quartil (Q3) e 1,5 amplitude interquartílica. Com exceção do $\mathrm{CN}$, os tratamentos foram submetidos à análise de variância, e as médias foram comparadas pelo teste de Duncan a $5 \%$ de probabilidade. Além disso, por meio de modelos regressivos e da correlação de Pearson (r), as relações entre as variáveis foram avaliadas com uso do software SAS (SAS Institute, Cary, EUA).

\section{Resultados e discussão}

A redução no preparo do solo e o controle de tráfego de máquinas e implementos resultaram em progressiva mudança no sistema poroso ao longo do tempo, sobretudo pela promoção da Pt e Ma e redução nos valores da relação Mi:Ma (Tabela 2).

Mudanças iniciais nesses atributos podem ser imperceptíveis com o menor tempo de implementação do SPD, como observado em Pt e Ma nas diferentes camadas em SPD1 e SPD3 (Tabela 2). Esses resultados corroboram os de Pauletto et al. (2005), que verificaram que 3 anos de adoção do SPD, em solo de várzea (Gleissolo Háplico), é pouco para que os efeitos 
no sistema poroso possam ser observados. Os autores sugerem um período maior que 5 anos para que se possam constatar os efeitos da implantação do SPD, o que condiz com os dados observados na Figura 1. Além disso, Pauletto et al. (2005) observaram valores de Mi entre 0,29 e 0,39, e de Mi:Ma entre 0,07:0,01 e 0,13:0,01, próximos aos obtidos neste estudo, que variaram de 0,32 a 0,40 e 0,04:0,01 a $0,28: 0,01 \mathrm{~m}^{3} \mathrm{~m}^{-3}$, respectivamente. As camadas superficiais sofreram efeito imediato dos sistemas de manejo, que alteraram, sobretudo, o sistema poroso do solo, com reduções na Mi e Mi:Ma em razão do aumento no tempo de implantação do SPD (Figura 1).

Os valores de $\mathrm{Ma}(\mathrm{r}=0,40 ; \mathrm{p}<0,01)$ e $\mathrm{Mi}(\mathrm{r}=0,80$; $\mathrm{p}<0,01)$ estiveram positivamente correlacionados com os de Pt (Tabela 3) e, embora tenham sido observadas reduções nos valores de Mi com o tempo de implantação, observou-se relação inversa entre Pt e Mi:Ma $(\mathrm{r}=-17 ; \mathrm{p}<0,01)$. Além de Mi:Ma, Rp $(\mathrm{r}=-0,50 ; \mathrm{p}<0,01)$ e Ds $(\mathrm{r}=-0,39 ; \mathrm{p}<0,01)$ também se correlacionaram negativamente com a Pt.

Palm et al. (2014) ressaltam a divergência sobre os efeitos do SPD e PC em diferentes camadas e atributos, principalmente sobre o volume de poros e Ds, que são variáveis inversamente relacionadas.

Valores de Rp variaram de 0,73 a 2,20 MPa, em que SPD1 foi a área de maior Rp, com 1,5 MPa a mais que o SPD7, em média (Tabela 4). Além disso, valores de Ds variaram entre 1,35 e 1,76 $\mathrm{Mg} \mathrm{ha}^{-1}$, também evidenciaram as diferenças entre SPD1 e SPD7. Moraes et al. (2014) e López-Garrido et al. (2014) relataram incremento da Ds em solo sob SPD, em comparação com solo sob PC. Schwen et al. (2011), ao avaliar o efeito de diferentes sistemas de manejo em um Chernossolo, obtiveram valores médios de $\mathrm{Pt}$ significativamente menores no SPD em comparação ao PC $\left(0,46\right.$ e $0,50 \mathrm{~m}^{3} \mathrm{~m}^{-3}$, respectivamente), bem como valores maiores de Ds $(1,44$ e 1,34 $\mathrm{Mg} \mathrm{m}^{-3}$ ). Os autores atribuíram essa diferença ao

Tabela 2. Média \pm erro-padrão da porosidade total, macroporosidade, microporosidade, relação Mi:Ma, em um Planossolo Háplico sob diferentes tempos de implantação do sistema plantio direto, bem como em campo não cultivado $(\mathrm{CN})^{(1)}$.

\begin{tabular}{|c|c|c|c|c|}
\hline \multirow[t]{2}{*}{ Tratamento } & Porosidade total & Macroporosidade (Ma) & Microporosidade (Mi) & Mi:Ma \\
\hline & & - $\left(\mathrm{m}^{3} \mathrm{~m}^{-3}\right)$ & & \\
\hline & \multicolumn{4}{|c|}{ Camada de 0,00 a $0,03 \mathrm{~m}$} \\
\hline SPD1 & $0,42 \pm 0,01 b$ & $0,01 \pm 0,00 \mathrm{~d}$ & $0,40 \pm 0,01 \mathrm{a}$ & $0,29 \pm 0,01 \mathrm{a}$ \\
\hline SPD3 & $0,42 \pm 0,01 \mathrm{ab}$ & $0,03 \pm 0,00 \mathrm{c}$ & $0,39 \pm 0,01 \mathrm{a}$ & $0,15 \pm 0,02 b$ \\
\hline SPD5 & $0,43 \pm 0,01 \mathrm{ab}$ & $0,04 \pm 0,00 \mathrm{~b}$ & $0,39 \pm 0,01 \mathrm{a}$ & $0,10 \pm 0,01 \mathrm{c}$ \\
\hline SPD7 & $0,45 \pm 0,01 \mathrm{a}$ & $0,06 \pm 0,00 \mathrm{a}$ & $0,38 \pm 0,01 \mathrm{a}$ & $0,06 \pm 0,00 \mathrm{~d}$ \\
\hline \multirow[t]{2}{*}{$\mathrm{CN}$} & $0,46 \pm 0,01$ & $0,09 \pm 0,01$ & $0,37 \pm 0,01$ & $0,05 \pm 0,01$ \\
\hline & \multicolumn{4}{|c|}{ Camada de 0,03 a $0,06 \mathrm{~m}$} \\
\hline SPD1 & $0,38 \pm 0,01 \mathrm{~b}$ & $0,02 \pm 0,00 \mathrm{c}$ & $0,36 \pm 0,01 \mathrm{a}$ & $0,19 \pm 0,01 \mathrm{a}$ \\
\hline SPD3 & $0,39 \pm 0,01 \mathrm{ab}$ & $0,03 \pm 0,00 \mathrm{~b}$ & $0,36 \pm 0,01 \mathrm{a}$ & $0,12 \pm 0,01 \mathrm{~b}$ \\
\hline SPD5 & $0,40 \pm 0,01 \mathrm{ab}$ & $0,04 \pm 0,00 \mathrm{~b}$ & $0,36 \pm 0,01 \mathrm{a}$ & $0,10 \pm 0,00 \mathrm{~b}$ \\
\hline SPD7 & $0,41 \pm 0,01 \mathrm{a}$ & $0,06 \pm 0,00 \mathrm{a}$ & $0,35 \pm 0,00 \mathrm{a}$ & $0,06 \pm 0,01 \mathrm{c}$ \\
\hline \multirow[t]{2}{*}{$\mathrm{CN}$} & $0,41 \pm 0,01$ & $0,08 \pm 0,01$ & $0,33 \pm 0,01$ & $0,05 \pm 0,01$ \\
\hline & \multicolumn{4}{|c|}{ Camada de 0,06 a $0,10 \mathrm{~m}$} \\
\hline SPD1 & $0,39 \pm 0,01 \mathrm{ab}$ & $0,02 \pm 0,00 \mathrm{~b}$ & $0,37 \pm 0,01 \mathrm{a}$ & $0,24 \pm 0,02 \mathrm{a}$ \\
\hline SPD3 & $0,37 \pm 0,01 \mathrm{~b}$ & $0,02 \pm 0,00 \mathrm{~b}$ & $0,35 \pm 0,01 \mathrm{ab}$ & $0,21 \pm 0,02 \mathrm{ab}$ \\
\hline SPD5 & $0,39 \pm 0,01 \mathrm{ab}$ & $0,02 \pm 0,00 \mathrm{~b}$ & $0,37 \pm 0,01 \mathrm{a}$ & $0,18 \pm 0,02 b$ \\
\hline SPD7 & $0,41 \pm 0,01 \mathrm{a}$ & $0,07 \pm 0,00 \mathrm{a}$ & $0,34 \pm 0,01 b$ & $0,04 \pm 0,00 \mathrm{c}$ \\
\hline \multirow[t]{2}{*}{$\mathrm{CN}$} & $0,42 \pm 0,01$ & $0,08 \pm 0,01$ & $0,33 \pm 0,00$ & $0,04 \pm 0,01$ \\
\hline & \multicolumn{4}{|c|}{ Camada de $0,10 \mathrm{~m}$ a $0,20 \mathrm{~m}$} \\
\hline SPD1 & $0,37 \pm 0,01 \mathrm{a}$ & $0,02 \pm 0,00 \mathrm{a}$ & $0,35 \pm 0,01 \mathrm{a}$ & $0,22 \pm 0,03 \mathrm{a}$ \\
\hline SPD3 & $0,37 \pm 0,01 \mathrm{a}$ & $0,02 \pm 0,00 \mathrm{a}$ & $0,35 \pm 0,01 \mathrm{a}$ & $0,17 \pm 0,01 \mathrm{ab}$ \\
\hline SPD5 & $0,37 \pm 0,01 \mathrm{a}$ & $0,03 \pm 0,00 \mathrm{a}$ & $0,34 \pm 0,01 \mathrm{a}$ & $0,14 \pm 0,01 \mathrm{bc}$ \\
\hline SPD7 & $0,37 \pm 0,01 \mathrm{a}$ & $0,04 \pm 0,00 \mathrm{a}$ & $0,33 \pm 0,01 \mathrm{a}$ & $0,09 \pm 0,01 \mathrm{c}$ \\
\hline $\mathrm{CN}$ & $0,38 \pm 0,01$ & $0,05 \pm 0,00$ & $0,32 \pm 0,01$ & $0,06 \pm 0,01$ \\
\hline
\end{tabular}

${ }^{(1)}$ Médias seguidas pela mesma letra nas colunas não diferem pelo teste de Duncan, a 5\% de probabilidade. SPD1, SPD3, SPD5 e SPD7 representam áreas com 1, 3, 5 e 7 anos de implantação do sistema plantio direto, respectivamente. 
efeito do adensamento natural promovido por 12 anos de adoção do SPD, sem revolvimento do solo.

Os valores de $\mathrm{Rp}$ foram menores no campo não cultivado $(\mathrm{CN})$, mas indicaram que os efeitos do PC sobre esse atributo podem ser mitigados com o tempo de implantação do SPD, sobretudo pela manutenção de resíduos orgânicos na superfície do solo, que favorece a estruturação do solo e sua qualidade física. As diferenças nesse atributo foram verificadas principalmente na camada de 0,00 a $0,03 \mathrm{~m}$. Já quanto ao DMP, observou-se que o SPD1 apresentou os menores diâmetros de agregados na camada de 0,00 a $0,03 \mathrm{~m}$ e 0,03 a $0,06 \mathrm{~m}$ (Tabela 4).

Karlen et al. (2013), ao avaliar solos cultivados continuamente com milho sob SPD, observaram $483 \mathrm{~g} \mathrm{~kg}^{-1}$ de macroagregados, em contraste com os
$298 \mathrm{~g} \mathrm{~kg}^{-1}$ observados em solo manejado com arado de aiveca, e os $383 \mathrm{~g} \mathrm{~kg}^{-1}$ com escarificador, na camada de 0,00 a $0,05 \mathrm{~m}$. Já na camada de 0,05 a $0,15 \mathrm{~m}$, os autores relataram $489 \mathrm{~g} \mathrm{~kg}^{-1}$ de macroagregados sob SPD, $352 \mathrm{~g} \mathrm{~kg}^{-1}$ sob arado de aiveca e $443 \mathrm{~g} \mathrm{~kg}^{-1}$ sob solo escarificado. Relataram ainda que operações de preparo afetam negativamente os teores de COT, ou de suas frações, ao longo do tempo.

Em SPD3, SPD5 e SPD7, foram observados valores elevados de macroagregados, que se distinguiram dos valores em SPD1 na camada de 0,00 a $0,03 \mathrm{~m}$, e evidenciaram efeito do tempo de implantação do SPD sobre o atributo. O maior tempo de implantação também aumentou o DMP dos agregados. Conforme Silva et al. (2008), o SPD com 8 anos é suficiente para promover melhorias na qualidade física. No entanto,
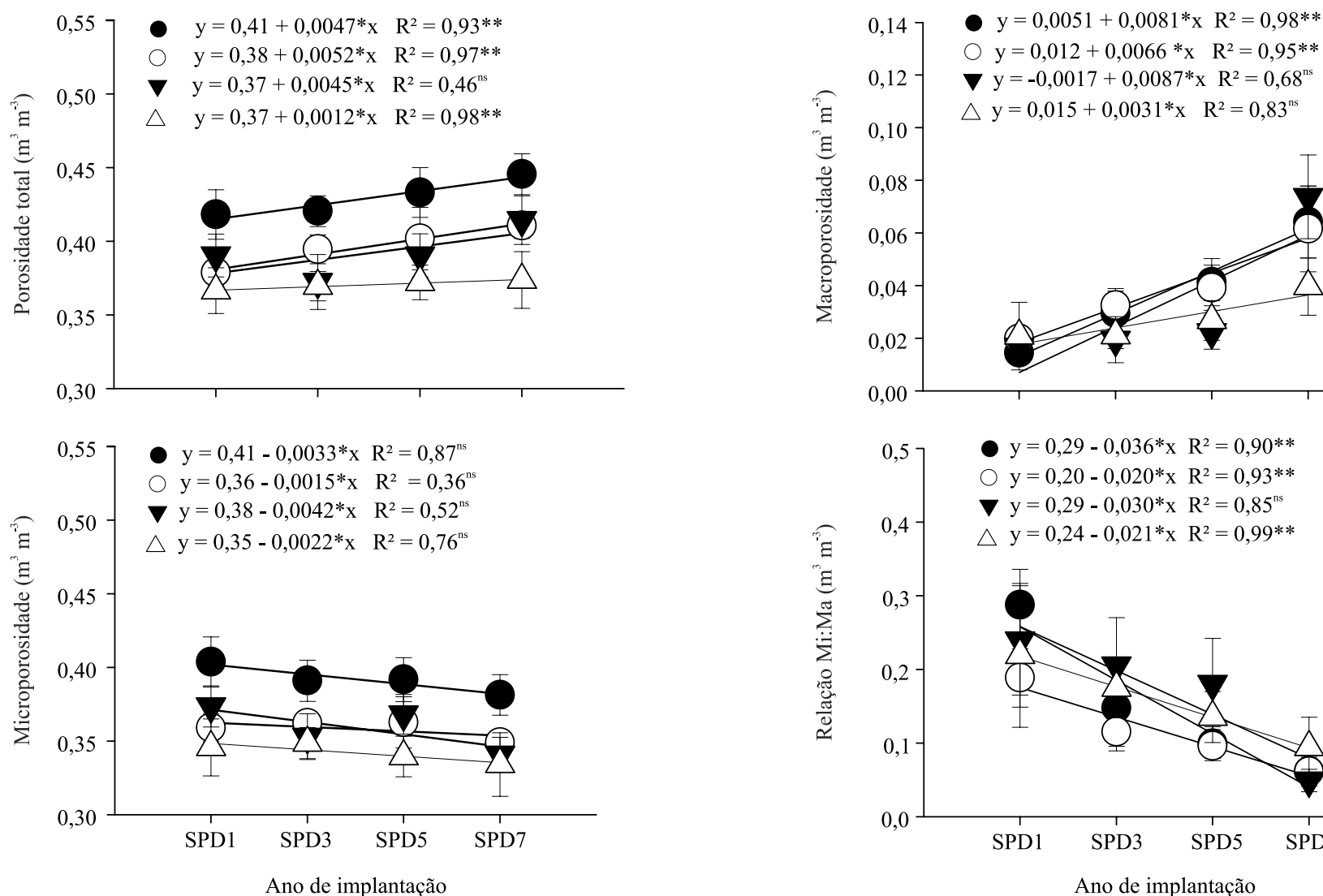

0,00 a $0,03 \mathrm{~m} \bigcirc 0,03$ a $0,06 \mathrm{~m}$
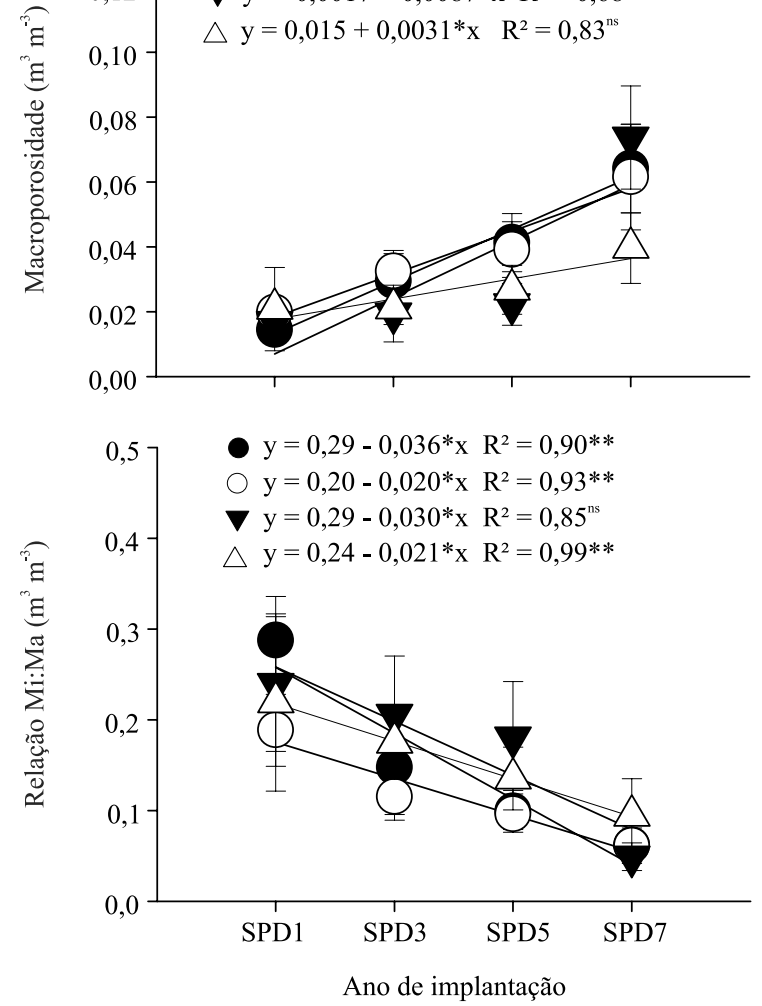

0,06 a $0,10 \mathrm{~m} \triangle 0,10$ a $0,20 \mathrm{~m}$

Figura 1. Porosidade total (Pt), macroporosidade (Ma), microporosidade (Mi) e relação Mi:Ma em um Planossolo Háplico sob diferentes tempos (T) de implantação do sistema plantio direto, nas diversas camadas do solo avaliadas. Barras verticais

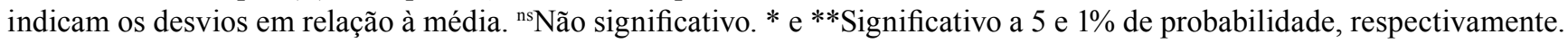
SPD1, SPD3, SPD5 e SPD7 representam áreas com 1, 3, 5 e 7 anos de implantação do sistema plantio direto, respectivamente. 
o presente trabalho, em solo de várzea, sugere que apenas 5 anos de implantação de SPD já podem evidenciar melhorias estruturais no solo.

De maneira geral, o COT aumentou nas camadas superficiais, com o tempo de implantação do SPD, mas

Tabela 3. Coeficientes de correlação de Pearson entre atributos físicos de um Planossolo Háplico, na camada de 0,00 a $0,20 \mathrm{~m}$, sob diferentes tempos de implantação do sistema plantio direto.

\begin{tabular}{lccccc}
\hline & $\mathrm{Ma}$ & $\mathrm{Mi}$ & $\mathrm{Mi}: \mathrm{Ma}$ & $\mathrm{Rp}$ & $\mathrm{Ds}$ \\
\hline $\mathrm{Pt}$ & $0,40^{* *}$ & $0,80^{* *}$ & $-0,17^{* *}$ & $-0,50^{* *}$ & $-0,39^{* *}$ \\
$\mathrm{Ma}$ & & $-0,15^{* *}$ & $-0,83^{* *}$ & $-0,40^{* *}$ & $-0,47^{* *}$ \\
$\mathrm{Mi}$ & & $0,30^{* *}$ & $-0,29 * *$ & $-0,16^{* *}$ \\
$\mathrm{Mi}: \mathrm{Ma}$ & & & & $0,32^{* *}$ & $0,37^{* *}$ \\
$\mathrm{Rp}$ & & & & & $0,45^{* *}$ \\
\hline
\end{tabular}

**Significativo a $1 \%$ de probabilidade $(\mathrm{n}=192)$. Pt: porosidade total $\left(\mathrm{m}^{3} \mathrm{~m}^{-3}\right)$; Ma: macroporosidade $\left(\mathrm{m}^{3} \mathrm{~m}^{-3}\right)$; Mi: microporosidade $\left(\mathrm{m}^{3} \mathrm{~m}^{-3}\right)$; Mi:Ma, Relação micro/macroporoso $\left(\mathrm{m}^{3} \mathrm{~m}^{-3}\right)$; Rp: resistência à penetração (MPa); Ds: densidade do solo ( $\left.\mathrm{Mg} \mathrm{m}^{-3}\right)$. diminuiu com o aumento da profundidade (Tabela 5). Foram observadas diferenças significativas na FLL, sobretudo em SPD7, em todas as camadas avaliadas. Além disso, em SPD, os fragmentos orgânicos no perfil do solo (FLL) que entram em processo de decomposição incorporaram MO à matriz do solo (FLO e FP) e reduziram a $\mathrm{Rp}$ e a Ds de forma cumulativa com o tempo, já que não houve revolvimento do solo que elevasse a oxidação da matéria orgânica, o que, consequentemente, favoreceu o aumento nos estoques de C (Figura 2), agregação, DMP e Pt.

Maiores teores de $\mathrm{C}$ estiveram associados à $\mathrm{FP}$, o que corrobora os resultados de Rosa et al. (2011), em Planossolo sob diferentes sistemas de manejo. Entretanto, as quantidades observadas nas frações apresentaram ordem de importância $\mathrm{FP}>\mathrm{FLL}>\mathrm{FLO}$ contrastante à encontrada pelos autores (FP $>$ FLO $>$ FLL). A quantidade de FLL e FLO pode variar com o tipo de solução e com a energia de dispersão do solo utilizados

Tabela 4. Média \pm erro-padrão de macroagregados, microagregados, diâmetro médio ponderado (DMP) dos agregados estáveis em água, resistência à penetração (Rp) e densidade (Ds) em Planossolo Háplico sob diferentes tempos de implantação do sistema plantio direto e em campo não cultivado $(\mathrm{CN})^{(1)}$.

\begin{tabular}{|c|c|c|c|c|c|}
\hline Tratamento & Macro & Micro & $\mathrm{DMP}(\mathrm{mm})$ & $\mathrm{Rp}(\mathrm{MPa})$ & Ds $\left(\mathrm{Mg} \mathrm{m}^{-3}\right)$ \\
\hline & 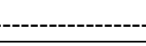 & - & & & \\
\hline & \multicolumn{5}{|c|}{ Camada de 0,00 a $0,03 \mathrm{~m}$} \\
\hline SPD1 & $524 \pm 1,23 b$ & $459 \pm 1,52 \mathrm{a}$ & $2,6 \pm 0,05 \mathrm{~b}$ & $1,12 \pm 0,11 \mathrm{a}$ & $1,62 \pm 0,03 \mathrm{a}$ \\
\hline SPD3 & $645 \pm 1,48 \mathrm{a}$ & $355 \pm 1,48 b$ & $3,6 \pm 0,16 \mathrm{a}$ & $0,90 \pm 0,12 \mathrm{ab}$ & $1,61 \pm 0,02 \mathrm{a}$ \\
\hline SPD5 & $634 \pm 1,06 \mathrm{a}$ & $366 \pm 1,06 b$ & $3,9 \pm 0,09 \mathrm{a}$ & $0,84 \pm 0,05 \mathrm{~b}$ & $1,56 \pm 0,03 \mathrm{a}$ \\
\hline SPD7 & $645 \pm 1,11 \mathrm{a}$ & $355 \pm 1,11 \mathrm{~b}$ & $3,9 \pm 0,13 \mathrm{a}$ & $0,80 \pm 0,05 \mathrm{~b}$ & $1,42 \pm 0,04 \mathrm{~b}$ \\
\hline \multirow[t]{2}{*}{$\mathrm{CN}$} & $697 \pm 1,13$ & $303 \pm 1,13$ & $4,2 \pm 0,04$ & $0,73 \pm 0,04$ & $1,35 \pm 0,02$ \\
\hline & \multicolumn{5}{|c|}{ Camada de 0,03 a $0,06 \mathrm{~m}$} \\
\hline SPD1 & $609 \pm 4,07 \mathrm{a}$ & $391 \pm 4,07 \mathrm{a}$ & $2,5 \pm 0,15 b$ & $1,44 \pm 0,10 \mathrm{a}$ & $1,74 \pm 0,02 \mathrm{a}$ \\
\hline SPD3 & $638 \pm 1,37 \mathrm{a}$ & $362 \pm 1,37 \mathrm{a}$ & $3,2 \pm 0,15 \mathrm{ab}$ & $1,12 \pm 0,06 \mathrm{~b}$ & $1,7 \pm 0,03 \mathrm{ab}$ \\
\hline SPD5 & $636 \pm 1,06 a$ & $363 \pm 1,06 a$ & $3,8 \pm 0,13 \mathrm{a}$ & $1,02 \pm 0,07 \mathrm{~b}$ & $1,64 \pm 0,02 \mathrm{bc}$ \\
\hline SPD7 & $624 \pm 1,42 \mathrm{a}$ & $376 \pm 1,42 \mathrm{a}$ & $3,5 \pm 0,11 \mathrm{a}$ & $0,97 \pm 0,04 \mathrm{~b}$ & $1,59 \pm 0,04 \mathrm{c}$ \\
\hline \multirow[t]{2}{*}{$\mathrm{CN}$} & $681 \pm 0,81$ & $319 \pm 0,81$ & $4,2 \pm 0,03$ & $0,84 \pm 0,06$ & $1,53 \pm 0,02$ \\
\hline & \multicolumn{5}{|c|}{ Camada de 0,06 a $0,10 \mathrm{~m}$} \\
\hline SPD1 & $549 \pm 1,05 a$ & $451 \pm 1,05 \mathrm{a}$ & $2,9 \pm 0,12 \mathrm{a}$ & $1,78 \pm 0,14 \mathrm{a}$ & $1,75 \pm 0,02 \mathrm{a}$ \\
\hline SPD3 & $619 \pm 2,14 \mathrm{a}$ & $381 \pm 2,14 \mathrm{a}$ & $3,1 \pm 0,16 \mathrm{a}$ & $1,35 \pm 0,06 \mathrm{~b}$ & $1,72 \pm 0,03 \mathrm{a}$ \\
\hline SPD5 & $623 \pm 1,11 \mathrm{a}$ & $377 \pm 1,11 \mathrm{a}$ & $3,1 \pm 0,14 \mathrm{a}$ & $1,3 \pm 0,08 \mathrm{~b}$ & $1,65 \pm 0,02 b$ \\
\hline SPD7 & $625 \pm 3,94 \mathrm{a}$ & $375 \pm 3,94 \mathrm{a}$ & $3,3 \pm 0,10 \mathrm{a}$ & $1,15 \pm 0,08 b$ & $1,63 \pm 0,02 b$ \\
\hline \multirow[t]{2}{*}{$\mathrm{CN}$} & $671 \pm 1,37$ & $329 \pm 1,37$ & $3,7 \pm 0,12$ & $0,89 \pm 0,04$ & $1,55 \pm 0,03$ \\
\hline & \multicolumn{5}{|c|}{ Camada de $0,10 \mathrm{~m}$ a $0,20 \mathrm{~m}$} \\
\hline SPD1 & $570 \pm 0,73 a$ & $430 \pm 0,73 a$ & $2,9 \pm 0,05 \mathrm{a}$ & $2,20 \pm 0,09 a$ & $1,76 \pm 0,01 \mathrm{a}$ \\
\hline SPD3 & $621 \pm 1,69 \mathrm{a}$ & $379 \pm 1,69 \mathrm{a}$ & $2,7 \pm 0,13 \mathrm{a}$ & $1,72 \pm 0,11 b$ & $1,71 \pm 0,03 \mathrm{ab}$ \\
\hline SPD5 & $605 \pm 1,41 \mathrm{a}$ & $395 \pm 1,41 \mathrm{a}$ & $3,3 \pm 0,18 \mathrm{a}$ & $1,56 \pm 0,08 b$ & $1,65 \pm 0,03 \mathrm{bc}$ \\
\hline SPD7 & $593 \pm 1,01 \mathrm{a}$ & $407 \pm 1,01 \mathrm{a}$ & $2,8 \pm 0,08 \mathrm{a}$ & $1,52 \pm 0,10 \mathrm{~b}$ & $1,59 \pm 0,04 \mathrm{c}$ \\
\hline $\mathrm{CN}$ & $625 \pm 1,60$ & $375 \pm 1,60$ & $3,3 \pm 0,12$ & $1,03 \pm 0,04$ & $1,59 \pm 0,02$ \\
\hline
\end{tabular}

${ }^{(1)}$ Médias seguidas pela mesma letra, nas colunas, não diferem pelo teste de Duncan, a 5\% de probabilidade. SPD1, SPD3, SPD5 e SPD7 representam áreas com 1,3, 5 e 7 anos de implantação do sistema plantio direto, respectivamente. 
nos procedimentos analíticos, com reflexos sobre os valores dessas frações (Conceição et al., 2008).

Segundo Roscoe et al. (2006), as diferenças entre os teores das frações da MOS refletem seus distintos graus de estabilidade, decorrentes de recalcitrância química (FLL), proteção física e oclusão (FLO) no interior dos agregados. Na FP, os três mecanismos podem atuar conjuntamente na manutenção do $\mathrm{C}$ (Nascimento et al., 2009), o que justifica os resultados observados para essa fração.

De modo geral, foram observadas diferenças estatísticas em IEC, LC, ILC e IMC, nas diferentes camadas avaliadas, exceto a de 0,06 a $0,10 \mathrm{~m}$ (Tabela 6). Maiores valores para os diferentes parâmetros foram verificados em SPD7, nas camadas de 0,00 a 0,03 m e 0,03

Tabela 5. Carbono orgânico total (COT), teor de carbono nas frações leve livre (FLL), leve oclusa (FLO) e pesada (FP) em Planossolo Háplico sob diferentes tempos de implantação do sistema plantio direto, bem como em campo não cultivado $(\mathrm{CN})^{(1)}$.

\begin{tabular}{|c|c|c|c|c|}
\hline \multirow{3}{*}{$\begin{array}{l}\text { Trata- } \\
\text { mento }\end{array}$} & COT & FLL & FLO & FP \\
\hline & & ----- $(\mathrm{g} \mathrm{l}$ & & \\
\hline & \multicolumn{4}{|c|}{ Camada de 0,00 a $0,03 \mathrm{~m}$} \\
\hline SPD1 & $4,81 \pm 0,05 \mathrm{c}$ & $0,74 \pm 0,10 \mathrm{c}$ & $0,45 \pm 0,01 \mathrm{~b}$ & $3,61 \pm 0,04 b$ \\
\hline SPD3 & $4,94 \pm 0,01 \mathrm{c}$ & $1,00 \pm 0,07 \mathrm{c}$ & $0,45 \pm 0,02 b$ & $3,48 \pm 0,08 b$ \\
\hline SPD5 & $8,61 \pm 0,11 b$ & $3,61 \pm 0,57 b$ & $0,49 \pm 0,02 \mathrm{ab}$ & $4,51 \pm 0,47 \mathrm{~b}$ \\
\hline SPD7 & $13,85 \pm 0,68 \mathrm{a}$ & $6,53 \pm 0,01 \mathrm{a}$ & $0,60 \pm 0,04 \mathrm{a}$ & $6,71 \pm 0,72 \mathrm{a}$ \\
\hline \multirow[t]{2}{*}{$\mathrm{CN}$} & $16,94 \pm 0,49$ & $4,20 \pm 0,17$ & $0,75 \pm 0,02$ & $11,99 \pm 0,30$ \\
\hline & \multicolumn{4}{|c|}{ Camada de 0,03 a $0,06 \mathrm{~m}$} \\
\hline SPD1 & $4,41 \pm 0,02 d$ & $0,53 \pm 0,03 \mathrm{~b}$ & $0,40 \pm 0,02 \mathrm{a}$ & $3,48 \pm 0,07 \mathrm{a}$ \\
\hline SPD3 & $4,64 \pm 0,00 \mathrm{c}$ & $0,81 \pm 0,15 b$ & $0,40 \pm 0,01 \mathrm{a}$ & $3,42 \pm 0,14 \mathrm{a}$ \\
\hline SPD5 & $7,45 \pm 0,07 b$ & $3,07 \pm 0,55 \mathrm{a}$ & $0,42 \pm 0,03 \mathrm{a}$ & $3,97 \pm 0,59 \mathrm{a}$ \\
\hline SPD7 & $8,39 \pm 0,02 \mathrm{a}$ & $3,93 \pm 0,12 \mathrm{a}$ & $0,45 \pm 0,03 \mathrm{a}$ & $4,00 \pm 0,11 \mathrm{a}$ \\
\hline \multirow[t]{2}{*}{$\mathrm{CN}$} & $16,14 \pm 0,07$ & $0,99 \pm 0,14$ & $0,91 \pm 0,06$ & $14,25 \pm 0,14$ \\
\hline & \multicolumn{4}{|c|}{ Camada de 0,06 a $0,10 \mathrm{~m}$} \\
\hline SPD1 & $4,27 \pm 0,04 \mathrm{c}$ & $0,68 \pm 0,11 b$ & $0,32 \pm 0,02 \mathrm{a}$ & $3,27 \pm 0,09 \mathrm{~b}$ \\
\hline SPD3 & $4,3 \pm 0,21 \mathrm{c}$ & $0,69 \pm 0,02 b$ & $0,32 \pm 0,02 \mathrm{a}$ & $3,28 \pm 0,17 b$ \\
\hline SPD5 & $7,07 \pm 0,02 b$ & $2,62 \pm 0,78 \mathrm{ab}$ & $0,39 \pm 0,06 \mathrm{a}$ & $4,05 \pm 0,82 b$ \\
\hline SPD7 & $10,67 \pm 0,02 \mathrm{a}$ & $3,31 \pm 0,15 \mathrm{a}$ & $0,42 \pm 0,00 \mathrm{a}$ & $6,94 \pm 0,13 \mathrm{a}$ \\
\hline \multirow[t]{2}{*}{$\mathrm{CN}$} & $10,81 \pm 2,45$ & $0,44 \pm 0,01$ & $0,75 \pm 0,00$ & $9,62 \pm 2,44$ \\
\hline & \multicolumn{4}{|c|}{ Camada de $0,10 \mathrm{~m}$ a $0,20 \mathrm{~m}$} \\
\hline SPD1 & $4,00 \pm 0,13 b$ & $0,49 \pm 0,02 \mathrm{a}$ & $0,26 \pm 0,00 \mathrm{a}$ & $3,24 \pm 0,11 b$ \\
\hline SPD3 & $3,94 \pm 0,00 \mathrm{~b}$ & $0,46 \pm 0,04 \mathrm{a}$ & $0,28 \pm 0,01 \mathrm{a}$ & $3,19 \pm 0,04 \mathrm{~b}$ \\
\hline SPD5 & $6,90 \pm 0,02 a$ & $0,61 \pm 0,02 \mathrm{a}$ & $0,31 \pm 0,06 \mathrm{a}$ & $5,98 \pm 0,06 \mathrm{a}$ \\
\hline SPD7 & $7,12 \pm 0,02 \mathrm{a}$ & $0,60 \pm 0,04 \mathrm{a}$ & $0,41 \pm 0,03 \mathrm{a}$ & $6,11 \pm 0,05 \mathrm{a}$ \\
\hline $\mathrm{CN}$ & $7,36 \pm 0,34$ & $0,48 \pm 0,11$ & $0,48 \pm 0,11$ & $6,41 \pm 0,34$ \\
\hline
\end{tabular}

${ }^{(1)}$ Médias \pm erro-padrão seguidas pela mesma letra, nas colunas, não diferem pelo teste de Duncan, a 5\% de probabilidade. SPD1, SPD3, SPD5 e SPD7 representam áreas com 1, 3, 5 e 7 anos de implantação do sistema plantio direto, respectivamente. a $0,06 \mathrm{~m}$, com especial contraste em relação ao SPD1, que apresentou os menores valores nessas camadas.

A razão entre o estoque de $\mathrm{C}$ nos diferentes tempos de implantação do SPD, em relação aos estoques em $\mathrm{CN}$, evidencia que o tempo de implantação do SPD favorece o acúmulo de $\mathrm{C}$ em todas as camadas. Entretanto, esse aumento nos estoques (IEC) não indica, necessariamente, permanência do $\mathrm{C}$ no solo. Valores de LC e ILC sustentam a afirmação de que a FLL e a FLO são mais suscetíveis às alterações provocadas pelo manejo e pela ação dos microrganismos do que o C presente na FP (Vieira et al., 2007).

Os valores de IEC, ILC e IMC foram maiores em SPD7, nas camadas de 0,00 a $0,03 \mathrm{~m}$ e 0,03 a $0,06 \mathrm{~m}$, o que demonstra que solos de várzea manejadas sob PC têm a sua qualidade recuperada a partir de 5 anos de implantação do SPD. Entretanto, ressalta-se que a eficiência de um sistema de manejo na recuperação da qualidade do solo depende, além das características desse manejo, do processo de degradação em que o solo se encontra. Esses resultados estão de acordo com os de Bona et al. (2006), em estudo da dinâmica da MOS em solo sob SPD e PC, e com os de Lanzanova et al. (2007), em estudos de dinâmica da MOS em áreas

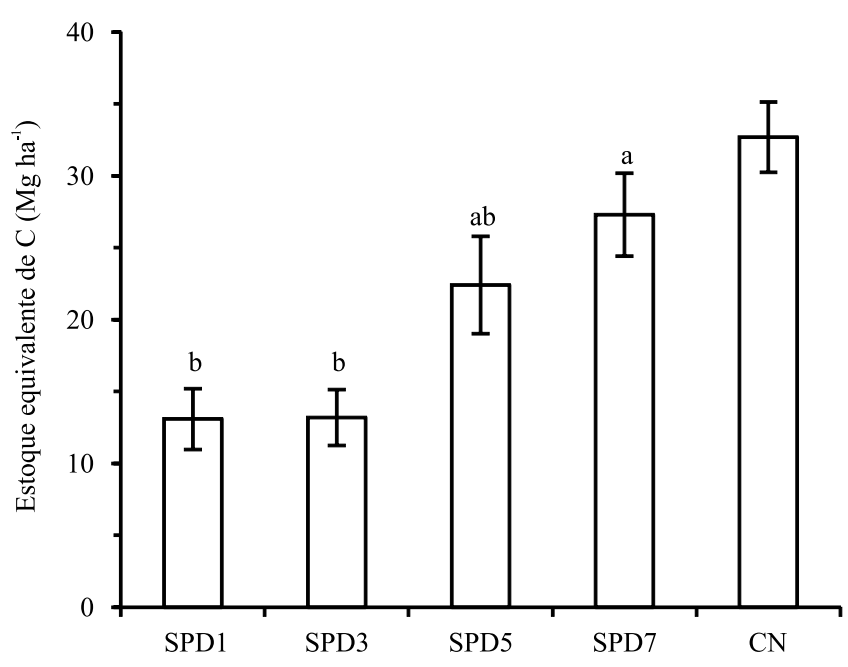

Figura 2. Estoque equivalente de carbono em Planossolo Háplico sob diferentes tempos de implantação do sistema plantio direto, e no campo não cultivado $(\mathrm{CN})$. Médias seguidas de letras iguais não diferem pelo teste de Duncan a $5 \%$ de probabilidade. Barras verticais indicam os desvios em relação à média. SPD1, SPD3, SPD5 e SPD7 representam áreas com 1, 3, 5 e 7 anos de implantação do sistema plantio direto, respectivamente. 
de integração lavoura-pecuária sob SPD. Os elevados valores de IMC em SPD5 e SPD7 mostram que esses sistemas foram eficientes na manutenção dos estoques de carbono e na melhoria da qualidade física do solo.

As bases da sustentabilidade agrícola podem ser observadas e promovidas a partir da adoção de práticas de manejo que favoreçam a agregação e a estruturação, e que reduzam a resistência à penetração e a densidade à medida que os teores e os estoques de carbono no solo são promovidos. Dessa forma, os resultados deste trabalho evidenciam que o SPD caracteriza-se como um sistema conservacionista também em solo de várzea, e que seus efeitos como promotor da qualidade do solo são destacados com o maior tempo de implantação.

Tabela 6. Índice de estoque de carbono (IEC), labilidade do carbono (LC), índice de labilidade do carbono (ILC) e índice de manejo de carbono (IMC) de um Planossolo Háplico eutrófico sob diferentes tempos de implantação do sistema plantio direto, bem como em campo não cultivado $(\mathrm{CN})^{(1)}$.

\begin{tabular}{|c|c|c|c|c|}
\hline Tratamento & IEC & LC & ILC & IMC \\
\hline & \multicolumn{4}{|c|}{ Camada de 0,00 a $0,03 \mathrm{~m}$} \\
\hline SPD1 & $0,34 \mathrm{c}$ & $0,33 b$ & $0,80 \mathrm{c}$ & $27,2 \mathrm{c}$ \\
\hline SPD3 & $0,35 \mathrm{c}$ & $0,42 b$ & $1,01 \mathrm{bc}$ & $35,3 \mathrm{c}$ \\
\hline SPD5 & $0,59 b$ & $0,95 \mathrm{ab}$ & $2,31 \mathrm{ab}$ & $136,2 b$ \\
\hline SPD7 & $0,86 \mathrm{a}$ & $1,08 \mathrm{a}$ & $2,62 \mathrm{a}$ & $225,1 \mathrm{a}$ \\
\hline \multirow[t]{2}{*}{$\mathrm{CN}$} & - & 0,41 & - & - \\
\hline & \multicolumn{4}{|c|}{ Camada de 0,03 a $0,06 \mathrm{~m}$} \\
\hline SPD1 & $0,31 \mathrm{c}$ & $0,27 \mathrm{~b}$ & $2,07 \mathrm{c}$ & $64,1 b$ \\
\hline SPD3 & $0,32 \mathrm{c}$ & $0,36 b$ & $2,76 \mathrm{bc}$ & $88,3 \mathrm{~b}$ \\
\hline SPD5 & $0,49 b$ & $0,94 \mathrm{ab}$ & $7,24 \mathrm{ab}$ & $354,7 \mathrm{a}$ \\
\hline SPD7 & $0,54 \mathrm{a}$ & $1,10 \mathrm{a}$ & $8,46 \mathrm{a}$ & $456,7 \mathrm{a}$ \\
\hline \multirow[t]{2}{*}{$\mathrm{CN}$} & - & 0,13 & - & - \\
\hline & \multicolumn{4}{|c|}{ Camada de 0,06 a $0,10 \mathrm{~m}$} \\
\hline SPD1 & $0,48 \mathrm{a}$ & $0,31 \mathrm{a}$ & $2,21 \mathrm{a}$ & $106,1 \mathrm{a}$ \\
\hline SPD3 & $0,47 \mathrm{a}$ & $0,31 \mathrm{a}$ & $2,21 \mathrm{a}$ & $103,1 \mathrm{a}$ \\
\hline SPD5 & $0,75 \mathrm{a}$ & $0,85 \mathrm{a}$ & $6,06 \mathrm{a}$ & $454,5 \mathrm{a}$ \\
\hline SPD7 & $1,10 \mathrm{a}$ & $0,54 \mathrm{a}$ & $3,84 \mathrm{a}$ & $422,4 \mathrm{a}$ \\
\hline \multirow[t]{2}{*}{$\mathrm{CN}$} & - & 0,14 & - & - \\
\hline & \multicolumn{4}{|c|}{ Camada de 0,10 a $0,20 \mathrm{~m}$} \\
\hline SPD1 & $0,60 \mathrm{a}$ & $0,23 \mathrm{a}$ & $1,55 \mathrm{a}$ & $93,3 \mathrm{a}$ \\
\hline SPD3 & $0,58 b$ & $0,23 \mathrm{a}$ & $1,55 \mathrm{a}$ & $89,9 \mathrm{a}$ \\
\hline SPD5 & $0,98 \mathrm{a}$ & $0,15 b$ & $1,00 \mathrm{a}$ & $98,0 \mathrm{a}$ \\
\hline SPD7 & $0,97 \mathrm{a}$ & $0,16 \mathrm{~b}$ & $1,06 \mathrm{a}$ & $102,7 \mathrm{a}$ \\
\hline $\mathrm{CN}$ & - & 0,15 & - & - \\
\hline
\end{tabular}

\section{Conclusões}

1. O maior tempo de implantação do sistema plantio direto reduz a densidade, a resistência do solo à penetração e a relação micro/macroporos, em razão do aumento na porosidade total e no diâmetro médio ponderado dos agregados.

2. Estoques de carbono orgânico total das frações leve livre, leve oclusa e pesada associam-se positivamente com o tempo de implantação do sistema plantio direto, na camada de 0,00 a $0,20 \mathrm{~m}$ de profundidade.

3. O sistema de plantio direto promove os índices de estoque, de labilidade e de manejo do carbono do solo.

4. Cinco anos de implantação do sistema plantio direto são suficientes para que se detectem incrementos na estruturação e na qualidade física do Planossolo avaliado.

\section{Agradecimentos}

À Coordenação de Aperfeiçoamento de Pessoal de Nível Superior (Capes), pela concessão da bolsa de pesquisa ao primeiro autor; à Universidade Federal de Pelotas (UFPel) e à Empresa Brasileira de Pesquisa Agropecuária, pelo apoio e estrutura técnico-pessoal.

\section{Referências}

BLAIR, G.J.; LEFROY, R.D.B.; LISLE, L. Soil carbon fractions based on their degree of oxidation, and the development of a carbon management index for agricultural systems. Australian Journal of Agricultural Research, v.46, p.1459-1460, 1995. DOI: 10.1071/AR9951459.

BONA, F.D. de; BAYER, C.; BERGAMASCHI, H.; DIECKOW, J. Carbono orgânico no solo em sistemas irrigados por aspersão sob plantio direto e preparo convencional. Revista Brasileira de Ciência do Solo, v.30, p.911-919, 2006. DOI: 10.1590/S010006832006000500017.

CLAESSEN, M.E.C. (Org.). Manual de métodos de análise de solo. 2.ed. rev. e atual. Rio de Janeiro: Embrapa-CNPS, 1997. $212 \mathrm{p}$.

CONCEIÇÃO, P.C.; BOENI, M.; DIECKOW, J.; BAYER, C.; MIELNICZUK, J. Fracionamento densimétrico com politungstato de sódio no estudo da proteção física da matéria orgânica em solos. Revista Brasileira de Ciência do Solo, v.32, p.541-549, 2008. DOI: 10.1590/S0100-06832008000200009.

CUNHA, N.G. da; COSTA, F.A. da. Solos da Estação Terras Baixas da Embrapa Clima Temperado. Pelotas: Embrapa Clima Temperado, 2013. 6p. (Embrapa Clima Temperado. Circular técnica, 152). 
GOMES, A. da S.; SILVA, C.A.S. da; PARFIT, J.M.B.; PAULETTO, E.A.; PINTO, L.F.S. Caracterização de indicadores da qualidade do solo, com ênfase às áreas de várzea do Rio Grande do Sul. Pelotas: Embrapa Clima Temperado, 2006. 40p. (Embrapa Clima Temperado. Documentos, 169).

IRGA. INSTITUTO RIO GRANDENSE DO ARROZ. Safra 2013/14 - amostragem da participação dos sistemas de cultivo. 2014. Disponível em: <http://www.irga.rs.gov.br/ upload/20140225133041sistema_cultivo_13_14_ii.pdf $>$. Acesso em: 9 out. 2014.

KARLEN, D.L.; CAMBARDELLA, C.A.; KOVAR, J.L.; COLVIN, T.S. Soil quality response to long-term tillage and crop rotation practices. Soil and Tillage Research, v.133, p.54-64, 2013. DOI: $10.1016 /$ j.still.2013.05.013.

KEMPER, W.D.; ROSENAU, R.C. Aggregate stability and size distribution. In: KLUTE, A. (Ed.). Methods of soil analysis. Part 1. Physical and mineralogical methods. $2^{\text {nd }}$ ed. Madison: American Society of Agronomy, Soil Science Society of America, 1986. p.425-441. DOI: 10.2136/sssabookser5.1.2ed.c17.

KLUTE, A. (Ed.). Methods of soil analysis. Part 1. Physical and mineralogical methods $2^{\text {nd }}$ ed. Madison: American Society of Agronomy, Soil Science Society of America, 1986. 1358p.

LANZANOVA, M.E.; NICOLOSO, R. da S.; LOVATO, T.; ELTZ, F.L.F.; AMADO, T.J.C.; REINERT, D.J. Atributos físicos do solo em sistema de integração lavoura-pecuária sob plantio direto. Revista Brasileira de Ciência do Solo, v.31, p.1131-1140, 2007. DOI: 10.1590/S0100-06832007000500028.

LISBOA, B.B.; VARGAS, L.K.; SILVEIRA, A.O. da; MARTINS, A.F.; SELBACH, P.A. Indicadores microbianos de qualidade do solo em diferentes sistemas de manejo. Revista Brasileira de Ciência do Solo, v.36, p.33-43, 2012. DOI: 10.1590/S010006832012000100004.

LÓPEZ-GARRIDO, R.; MADEJÓN, E.; LEÓN-CAMACHO, M.; GIRÓN, I.; MORENO, F.; MURILLO, J.M. Reduced tillage as an alternative to no-tillage under Mediterranean conditions: a case study. Soil and Tillage Research, v.140, p.40-47, 2014. DOI: 10.1016/j.still.2014.02.008.

MANUAL de adubação e de calagem para os Estados do Rio Grande do Sul e de Santa Catarina. 10.ed. Porto Alegre: Sociedade Brasileira de Ciência do Solo, 2004. 400p.

MORAES, M.T. de; DEBIASI, H.; CARLESSO, R.; FRANCHINI, J.C.; SILVA, V.R. da. Critical limits of soil penetration resistance in a rhodic Eutrudox. Revista Brasileira de Ciência do Solo, v.38, p.288-298, 2014. DOI: 10.1590/S010006832014000100029.

NASCIMENTO, P.C. do; BAYER, C.; NETTO, L. de F. da S.; VIAN, A.C.; VIEIRO, F.; MACEDO, V.R.M.; MARCOLIN, É. Sistemas de manejo e a matéria orgânica de solo de várzea com cultivo de arroz. Revista Brasileira de Ciência do Solo, v.33, p.1821-1827, 2009. DOI: 10.1590/S0100-06832009000600030.

PALM, C.; BLANCO-CANQUI, H.; DECLERCK, F.; GATERE, L.; GRACE, P. Conservation agriculture and ecosystem services: an overview. Agriculture, Ecosystems and Environment, v.187, p.87-105, 2014. DOI: 10.1016/j.agee.2013.10.010.
PALMEIRA, P. R. T.; PAULETTO, E. A.; TEIXEIRA, C. F. A.; GOMES, A. S.; SILVA, J. B. da. Agregação de um Planossolo submetido a diferentes sistemas de cultivo. Revista Brasileira de Ciência do Solo, v.23, p.189-195, 1999. DOI: 10.1590/S010006831999000200001.

PAULETTO, E.A.; BORGES, J.R.; SOUSA, R.O. de; PINTO, L.F.S.; SILVA, J.B. da; LEITZKE, V.W. Avaliação da densidade e da porosidade de um Gleissolo submetido a diferentes sistemas de cultivo e diferentes culturas. Revista Brasileira de Agrociência, v.11, p.207-210, 2005.

ROSA, C.M. da; CASTILHOS, R.M.V.; PAULETTO, E.A.; PILLON, C.N.; LEAL, O. dos A. Conteúdo de carbono orgânico em Planossolo Háplico sob sistemas de manejo do arroz irrigado. Revista Brasileira de Ciência do Solo, v.35, p.1769-1776, 2011. DOI: $10.1590 / \mathrm{S} 0100-06832011000500031$.

ROSCOE, R.; MADARI, B.E.; MACHADO, P.L.O.A. Fracionamento físico do solo na obtenção de compartimentos mensuráveis para uso em simuladores da dinâmica da matéria orgânica. In: ROSCOE, R.; MERCANTE, F.M.; SALTON, J.C. (Ed.). Dinâmica da matéria orgânica do solo em sistemas conservacionistas: modelagem matemática e métodos auxiliares. Dourados: Embrapa Agropecuária Oeste, 2006. p.107-132.

SCHWEN, A.; BODNER, G.; SCHOLL, P.; BUCHAN, G.D.; LOISKANDL, W. Temporal dynamics of soil hydraulic properties and the water-conducting porosity under different tillage. Soil and Tillage Research, v.113, p.89-98, 2011. DOI: 10.1016/j. still.2011.02.005.

SILVA, F. de F. da; FREDDI, O. da S.; CENTURION, J.F.; ARATANI, R.G.; ANDRIOLI, F.F.; ANDRIOLI, I. Propriedades físicas de um Latossolo Vermelho cultivado no sistema plantio direto. Irriga, v.13, p.191-204, 2008.

SISTI, C.P.J.; SANTOS, H.P. dos; KOHHANN, R.; ALVES, B.J.R.; URQUIAGA, S.; BODDEY, R.M. Change in carbon and nitrogen stocks in soil under 13 years of conventional or zero tillage in southern Brazil. Soil and Tillage Research, v.76, p.3958, 2004. DOI: 10.1016/j.still.2003.08.007.

TISDALL, J.M.; OADES, J.M. Organic matter and water-stable aggregates in soils. Journal of Soil Science, v.33, p.141-163, 1982. DOI: 10.1111/j.1365-2389.1982.tb01755.x.

TORMENA, C.A.; ARAÚJO, M.A.; FIDALSKI, J.; COSTA, J.M. da. Variação temporal do intervalo hídrico ótimo de um Latossolo Vermelho distroférrico sob sistemas de plantio direto. Revista Brasileira de Ciência do Solo, v.31, p.211-219, 2007. DOI: 10.1590/S0100-06832007000200003.

VELDKAMP, E. Organic carbon turnover in three tropical soils under pasture after deforestation. Soil Science Society of America Journal, v.58, p.175-180, 1994. DOI: 10.2136/ sssaj1994.03615995005800010025x.

VERNETTI JUNIOR, F. de J.; GOMES, A. da S.; SCHUCH, L.O.B. Sucessão de culturas em solos de várzea implantadas nos sistemas plantio direto e convencional. Revista Agrociência, v.15, p.37-42, 2009. DOI: 10.18539/cast.v15i1-4.1984.

VIEIRA, F.C.B.; BAYER, C.; ZANATTA, J.A.; DIECKOW, J.; MIELNICZUK, J.; HE, Z. L. Carbon management index 
based on physical fractionation of soil organic matter in an Acrisol under long-term no-till cropping systems. Soil Tillage and Research, v.96, p.195-204, 2007. DOI: 10.1016/j. still.2007.06.007.
YODER, R.E. A direct method of aggregate analysis of soil and a study of the physical nature of erosion losses. Journal of the American Society of Agronomy, v.28, p.337-351, 1936. DOI: 10.2134/agronj1936.00021962002800050001x.

$\overline{\text { Recebido em } 14 \text { de julho de } 2015 \text { e aprovado em } 16 \text { de fevereiro de } 2016}$ 